\title{
A feasibility study of transurethral resection followed by radiotherapy combined with concurrent twice-weekly gemcitabine in the treatment of invasive bladder carcinoma
}

\author{
Dalia Osama ${ }^{1}$, Mohamed Shalaby ${ }^{2}$, Mohamed Abdelhamed Aboziada ${ }^{1}$ \\ 1. Departments of Radiotherapy, South Egypt Cancer Institute, Assiut University, Egypt. 2. Urology Surgery, Faculty of \\ Medicine, Assiut University, Egypt
}

Correspondence: Mohamed Abdelhamed Aboziada. Address: Department of radiation Oncology, University of Assiut, South Egypt Cancer Institute, El-Methak St., Assiut, Egypt. E-mail: maboziada70@yahoo.com

Received: January 22, $2013 \quad$ Accepted: February 25, 2013

Online Published: March 11, 2013

DOI : $10.5430 /$ jst.v3n3p1

URL: http://dx.doi.org/10.5430/jst.v3n3p1

\section{Abstract}

Introduction and Aim: Radical cystectomy is the traditional treatment of cancer bladder; however, bladder preservation in selective patients is beneficial. To evaluate the efficacy of transurethral resection plus chemo-radiation in achieving bladder preservation, using conformal radiotherapy and twice weekly Gemcitabine.

Patients and Methods: Thirty seven patients with good performance status, and maximum trans-urethral resection received $46 \mathrm{GY} / 23$ fractions with twice weekly Gemcitabine $30 \mathrm{mg} / \mathrm{m}^{2}$. Evaluation was done after 2 weeks by cystoscopy and biopsy from the tumor bed. Patients who had complete response (CR) subjected for phase II $20 \mathrm{GY} / 10$ fractions $/ 2$ weeks with twice weekly Gemcitabine $30 \mathrm{mg} / \mathrm{m}^{2}$. However, patients who had incomplete response subjected for radical cystectomy.

Results: Thirty two (86.4\%) patients had CR. The treatment schedule was tolerable and was associated with moderate toxicity that was easily treated. Six patients developed G3 toxicity that required treatment interruption until improvement. After 2 years of follow up, 29 patients achieved good local control with 2 years local recurrence free survival was $79 \%$. The 2 years overall survival and bladder intact survival was $70 \%$ and $69 \%$ respectively.

Conclusions: Tri-modality bladder-sparing approach consists of transurethral resection, chemotherapy twice weekly Gemcitabine and radiotherapy is well tolerated, with high rate of bladder preservation. This approach may be considered as a reasonable alternative to cystectomy in the proper selected patients.

\section{Key words}

Bladder, Gemcitabine, Radiotherapy

\section{I ntroduction}

Carcinoma of the urinary bladder is diagnosed in approximately 71,000 people each year in the United States, making it the fourth most common cancer in men $(53,000)$ and ninth most common cancer in women $(18,000)^{[1]}$. Surgery achieves an excellent local control (82\%-92\%) with a peri-operative mortality less than $5 \%$, but it has a complication rate ranging 
from $25 \%$ - 40\%. It carries significant physical, sexual, and psychological morbidity like incontinence in $20 \%-50 \%$ of patients, impotence in 40\%-70\%, and an overall recurrence rate of $25 \%-40 \%$. Five-year specific and overall survival rates are $68 \%$ and $40 \%-60 \%$, and that probably dependent on the existence of micro metastases at time of diagnosis ${ }^{[2]}$.

Trimodality bladder-sparing approaches consisting of transurethral resection (TUR), chemotherapy and radiotherapy (RT) yield overall survival rates comparable with those of radical cystectomy series (50\%-70\% at 5 years), while preserving the native bladder in $40 \%-60 \%$ of muscle-invasive bladder cancer patients, contributing to an improvement in quality of life for such patients ${ }^{[3-5]}$.

Gemcitabine is active in bladder cancer and used in combination with platinum as a standard of care in the neoadjuvant and metastatic settings ${ }^{[6,7]}$. Studies have also shown that gemcitabine is a potent radiosensetizer, and its efficacy has been demonstrated in various cancer cell lines, including lines derived from bladder tumors ${ }^{[8-10]}$. Twice weekly gemcitabine with concurrent radiotherapy at 2 Gy per day is well tolerated and have high rate of bladder preservation in the selected group of patients ${ }^{[11]}$.

\section{Aim of the work}

- Our primary end points are to investigate the tolerance and toxicity of a modified concurrent chemo radiation protocol for patients with invasive bladder cancer and to evaluate its efficacy in achieving a complete response.

- The secondary end point is to estimate local control, disease free survival, overall survival, and bladder preservation rate.

\section{Patients and methods}

This study had been conducted on 37 patients, from January 2009 to May 2012 and approved by our institute ethics committee.

\subsection{Eligibility criteria}

Transitional cell carcinoma of the bladder with histological evidence was proven. Clinical stages T2-T3b, N0, M0, (High grade T1 was included), based upon the results of the cystoscopy, TUR, and radiographic investigation. Patients had undergone a maximal transurethral resection of the bladder tumor. Performance status $\leqslant 2$ score according to Eastern Cooperative Oncology Group (ECOG) performance scale. Hemoglobin $>10 \mathrm{gm} / \mathrm{dL}$, WBC $>4000 / \mathrm{mL}$, an absolute neutrophil count of $>1800 / \mathrm{mL}$, a platelet count of $>100,000 / \mathrm{mL}$, a serum creatinine of $1.5 \mathrm{mg} \%$ or less, a serum bilirubin of $2.0 \mathrm{mg} \%$ or less and a creatinine clearance of $60 \mathrm{~mL} / \mathrm{min}$ or greater.

\subsection{I neligibility criteria}

Patients were ineligible if they have multicentric tumors and received intra-vasical BCG, chemotherapy or pelvic irradiation and distant metastasis.

\subsection{Treatment protocol}

It was begun within 4-6 weeks after the maximal TUR, and all patients received combined chemo-radiation in two phases. Radiotherapy delivered in conventional fractionation; phase I (46GY/23 fractions/4.5weeks), then phase II; 20GY/10 fractions $/ 2$ weeks for complete responder. Gemcitabine delivered $30 \mathrm{mg} / \mathrm{m}^{2}$ twice weekly as a 30 -mimute infusion within 2 hours before radiation therapy. 


\subsection{Radiotherapy}

Phase I was delivered in 2 steps. Two steps, included bladder phase 20Gy/10 fractions/2 weeks and pelvic phase 26Gy/13 fractions/2.6 weeks. After 2 weeks, evaluation was done by cystoscopic examination and tumor bed biopsy. Patients who achieved complete response (CR) proceed for phase II. Patients who achieved incomplete response proceed for radical cystectomy. Phase II started 1 week after cystoscopic evaluation, and included the entire pelvic irradiation for 2 weeks.

\subsection{Radiotherapy technique}

Position: Patients were treated in supine position with empty bladder on suitable head rest with under knee support, prior to simulation, and before every session of treatment. The rectum should be as empty as possible before simulation (may use enema).

Localization: CT simulator was done; cuts started from L4 till the upper femur every $0.5 \mathrm{~cm}$, used for localization and determination of target volume, and organs at risk; rectum, small intestine, bilateral femoral head. CT data transferred to the computer treatment planning system ( $\mathrm{XiO} 4.2)$.

Bladder target volume: This volume included the whole bladder volume plus 1-1.5 $\mathrm{cm}$ margin beyond. This volume shaped using cerrobend blocks.

Pelvic target volume: This volume included the bladder, the prostate and the prostatic urethra (in men) and the regional lymph nodes (perivesical lymph node, distal hypogastric, external iliac vessels and those within the obturator space). The field margins of planning target volume (PTV) in the cranio-caudal dimension extended from S1-S2 junction to the lower pole of the obturator foramen. In the anterior and posterior pelvic field, PTV widths extended $1.0 \mathrm{~cm}$ lateral to the bony margin of the pelvis at its widest point. For the two parallel-opposed fields, the anterior boundary of the PTV will be 1.5 $\mathrm{cm}$ anterior to the most anterior portion of the bladder. Posterior, the PTV extended at least $1.5 \mathrm{~cm}$ posterior to the most posterior portion of the bladder. This volume shaped by using cerrobend blocks.

\subsection{Radiation dose and energy}

The radiotherapy course deliverd 46 Gy to the pelvic fields, 66 Gy to whole bladder. All doses prescribed at the iso center, using linear accelerator 6-15 MV. Total dose did not exceed $45 \mathrm{~Gy}$ and $50 \mathrm{~Gy}$ to the femoral heads and the posterior rectal wall respectively.

\subsection{Follow up of patients during the treatment}

During treatment, we evaluated patients weekly and monitor toxicity. We performed complete blood counts, including differential and platelets before each administration of Gemcitabine. Additional laboratory investigations every 3 weeks included electrolytes, liver function, and kidney function.

\subsection{Follow up after treatment}

- Physical examination every 1 month and then every 3 months after 6months.

- Laboratory investigation: (complete blood count, urea, creatinine, liver function, and urine analysis during every visit).

- Cystoscopic evaluation was done every three months in the first year, and every six months during the second year, and annually after the second year.

- Chest X ray and CT pelvi-abdomen done every 6 month during first year then annually after. 


\subsection{Statistical method of analysis}

Statistical analysis of data was done by the statistical package for the social science (SPSS) version ${ }^{[18]}$. Kaplan Meier test used for survival analysis, and Log rank test was used to evaluate the significant differences between variables. Chi-square test was used to evaluate the relation between variables and treatment response. $P$-value was double sided and considered significant if was $\leqslant 0.05$. Acute and chronic toxicities from radiotherapy and chemotherapy were reported according to Common Toxicity Criteria for Adverse Events (CTCAE) version 3.0 ${ }^{[12]}$.

Table 1. Patient's characteristics

\begin{tabular}{llll}
\hline Variable & & Number & Percentage (\%) \\
\hline \multirow{2}{*}{ Age group } & $<50$ & 8 & 22 \\
& $\geq 50$ & 29 & 78 \\
Performance status (PS) & PS1 & 29 & 78 \\
& PS2 & 8 & 22 \\
Bilharizial & YES & 28 & 76 \\
& NO & 9 & 24 \\
Smoking & YES & 24 & 65 \\
& NO & 13 & 35 \\
Hydronephrosis & YES & 4 & 11 \\
& NO & 33 & 89 \\
\multirow{2}{*}{ T Stage } & T1 & 4 & 11 \\
& T2 & 25 & 68 \\
Grade & T3 & 8 & 22 \\
\multirow{2}{*}{ TURT } & Low & 8 & 22 \\
& High & 29 & 78 \\
\hline
\end{tabular}

TURT, trans urethral resection of tumor

\section{Results}

The 37 patients completed the study treatment protocol and follow up schedule. General patient's characteristics were listed in Table 1. We assessed the response to chemo-radiation 2 weeks after phase I. Thirty two patients (86.5\%) had no disease (complete response) and 5 patients (13.5\%) had incomplete response. Out of the 5 patients who had incomplete response 2 patients had minimal residuals therefore, they continued in phase two. Also, one patient who had incomplete response had disease progression, and radical cystectomy was carried out. The last two patients had developed distant metastasis and referred to chemotherapy. Hydronephrosis was the only significant prognostic factor affecting the response (Table 2).

The median follow up was 2 years. The local recurrence free survival was $79 \%$. Two years cystectomy free survival was $83 \%$. The 2 years disease free survival was $50 \%$. Nineteen out of 37 patients developed either local recurrence (LR) and or distant metastasis (DM). Five patients developed LR, 12 patients developed DM, and 2 patients developed both LR \& DM. Distant metastasis rate was $37.8 \%$ (14/37). Sites of distant metastasis were in bone (38\%) and pelvic lymph nodes $(23.8 \%)$ followed by liver (19\%), lung (14.2\%) and 1 patient had brain secondary. The status of complete resection and the presence of hydronephrosis were the significant factors affecting the disease free survival. The 2 years overall survival and bladder intact survival was $70 \%$ and $69 \%$ respectively. 
Table 2. Factors affecting the response

\begin{tabular}{|c|c|c|c|c|}
\hline Variables & & Number & Complete response (\%) & $P$ \\
\hline Performance & PS1 & 29 & $25(86 \%)$ & \multirow{3}{*}{0.61} \\
\hline \multirow[t]{2}{*}{ Status } & PS2 & 8 & $7(88 \%)$ & \\
\hline & $\mathrm{T} 1$ & 4 & $4(100 \%)$ & \\
\hline \multirow{2}{*}{ T Stage } & $\mathrm{T} 2$ & 25 & $21(84 \%)$ & \multirow{2}{*}{0.46} \\
\hline & $\mathrm{T} 3$ & 8 & $7(88 \%)$ & \\
\hline \multirow{2}{*}{ Bilharzial } & Yes & 28 & $26(93 \%)$ & \multirow{2}{*}{0.16} \\
\hline & No & 9 & $6(67 \%)$ & \\
\hline \multirow{2}{*}{ TURT } & Complete & 28 & $25(89 \%)$ & \multirow{2}{*}{0.20} \\
\hline & Incomplete & 9 & $7(78 \%)$ & \\
\hline \multirow{2}{*}{ Hydronephrosis } & Yes & 4 & $2(50 \%)$ & \multirow{2}{*}{0.006} \\
\hline & No & 33 & $30(91 \%)$ & \\
\hline \multirow{2}{*}{ Grade } & Low & 8 & $7(88 \%)$ & \multirow{2}{*}{0.19} \\
\hline & High & 29 & $25(86 \%)$ & \\
\hline \multirow{2}{*}{ Treatment interruption } & No & 27 & $22(81 \%)$ & \multirow{2}{*}{0.54} \\
\hline & Yes & 10 & $10(100 \%)$ & \\
\hline
\end{tabular}

TURT, trans urethral resection of tumor.

Gemcitabine was chosen both because of its potent radiosensitizing properties and because of its more favorable toxicity profile. The acute adverse effects of grade 3 and higher were neutropenia in 3/37, thrombocytopenia in $3 / 37$, cystitis in $1 / 37$, and hepatic toxicity in $2 / 37$. The majority of patients had mild to moderate effects that improved with supportive treatment, and did not require treatment interruption, or major intervention (Table 3). Late reactions were observed in the bladder grade I (42.6\%), grade 2 (33.3\%), grade 3 (2.7\%) followed by the rectum grade1 (21.4\%).

Table 3. Early Toxicity

\begin{tabular}{lllllll}
\hline & G0 & G1 & G2 & G3 & G4 & Total \\
\hline Hg & $10(70 \%)$ & $9(24 \%)$ & $2(5 \%)$ & 0 & 0 & $11(30 \%)$ \\
WBCs & $33(89 \%)$ & $1(3 \%)$ & $1(3 \%)$ & $2(5 \%)$ & 0 & $4(11 \%)$ \\
Neutrophil & $33(89 \%)$ & $1(3 \%)$ & 0 & $2(5 \%)$ & $1(3 \%)$ & $4(11 \%)$ \\
Platelet & $27(73 \%)$ & $5(14 \%)$ & $2(5 \%)$ & $3(8 \%)$ & 0 & $10(27 \%)$ \\
Fatigue & $8(22 \%)$ & $14(38 \%)$ & $12(32 \%)$ & $3(8 \%)$ & 0 & $29(78 \%)$ \\
Bladder & $1(3 \%)$ & $17(46 \%)$ & $18(49 \%)$ & $1(3 \%)$ & 0 & $36(97 \%)$ \\
Rectum & $6(16 \%)$ & $23(62 \%)$ & $8(22 \%)$ & 0 & 0 & $31(84 \%)$ \\
Liver & 0 & $1(3 \%)$ & $1(3 \%)$ & $2(5 \%)$ & 0 & $4(11 \%)$ \\
\hline
\end{tabular}

\section{Discussion}

The most important points in this study were the high incidence of complete response, and the tolerability to the treatment protocol. However, there is also high incidence of distant failure rather than local failure.

In the present study, after phase 1, complete remission (CR) was $86 \%$. Those results matched with the results of Elizabeth et al ${ }^{[11]}$ who reported CR in $87 \%$. Kaufman et al ${ }^{[13]}$ reported CR in $81 \%$, and Hamza et al ${ }^{[14]}$ reported $80 \%$ CR. However, the response was higher than Donald et al ${ }^{[15]}$ in RTOG 95-06 and Aboziada et al ${ }^{[16]}$ who reported CR in $67 \%$ and $60 \%$ respectively. In the Massachusetts General Hospital (MGH) series of 190 patients between 1986 and 1998 treated by trimodality therapy, $63 \%$ exhibited a $\mathrm{CR}^{[17]}$. 
In the present study, prognostic factor which affects $\mathrm{CR}$ after phase 1 was the presence of hydronephrosis, comparable with Rincon et al ${ }^{[2]}$. In the MGH series ${ }^{[17]}, 27$ of 190 patients had hydronephrosis initially, and their complete response rate was $37 \%$, compared to $68 \%$ in patients without hydronephrosis $(p=0.002)$. Other factors like early stage and maximum TURBT are significantly affecting the response ${ }^{[13,16-20]}$. Grade of tumor is reflecting the behavior of disease. It was significant in some trials ${ }^{[16,18]}$.

Figure1. Overall survival

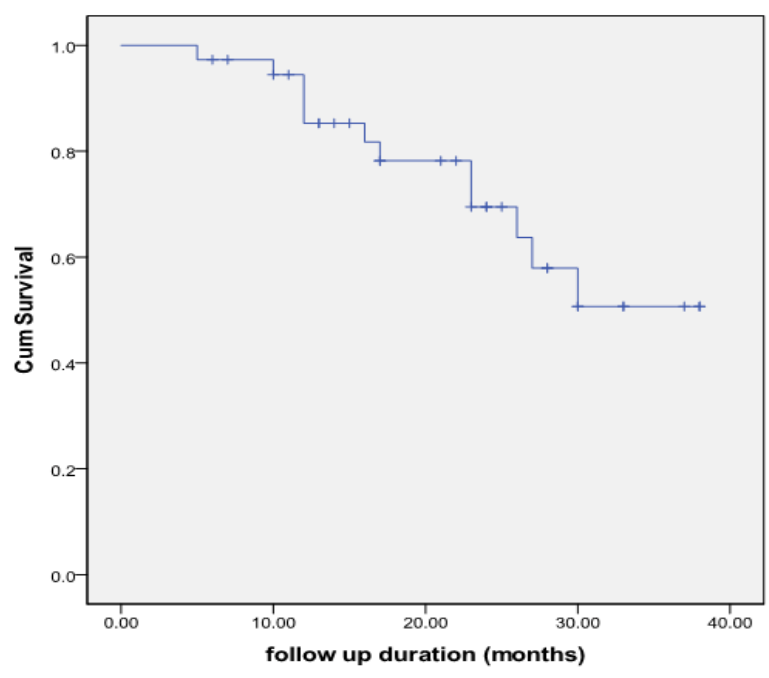

The radiotherapy used in this trial was delivered with conformal technique with an appropriate margin for set up uncertainties. We started with small volume followed by large field. This was chosen to limit the dose to small bowel facilitating salvage surgical management and to allow neobladder reconstruction, if necessary.

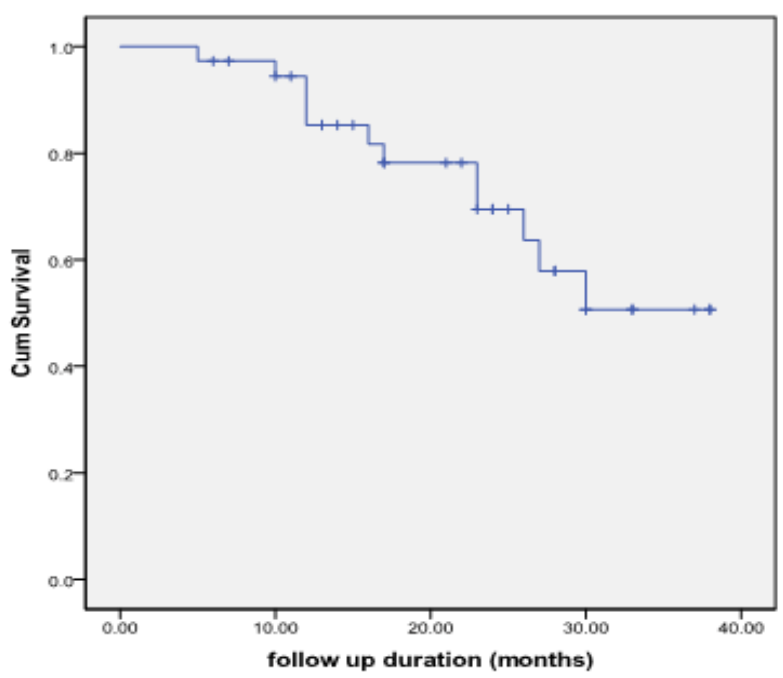

Figure 2. Bladder intact survival

For all patients, the overall survival rate at 2 years was 70\%, which comparable to that reported by most clinical trials. Choudhury et al ${ }^{[4]}$ who reported OAS $75 \%$ at 3 years, Cheon et al ${ }^{[21]}$ who reported $74 \%$ at 2 years and Lin et al ${ }^{[22]}$ who reported $77 \%$ at 3 years. However, Donald et al ${ }^{[15]}$ reported OAS at 3 years $83 \%$, that mostly due to more aggressive protocol including adjuvant chemotherapy (cisplatin and $5 \mathrm{FU}$ ). DFS at 2 years was $50 \%$, includes $22 \%$ local failure and 
$37 \%$ distant failure. These results are comparable to Lin et al ${ }^{[22]}$ who reported DFS $54 \%$ at 3 years. Langsenhner et al ${ }^{[23]}$ and Maarouf et al ${ }^{[24]}$ reported $40 \%$ at 3 years and $39 \%$ at 1 year respectively. However those are lower than it was reported by Rincon et al ${ }^{[2]}$ who reported PFS $69 \%$ at 3 years, but their protocol was used adjuvant 2-4 cycle of chemotherapy, (taxol, methotrexate, $5 \mathrm{FU}$, and cisplatin). Kevin et al ${ }^{[25]}$ reported the 5 -year actuarial OS $76 \%$, and DSS $82 \%$.

A meta-analysis of randomized studies showed survival benefits of cisplatin-containing systemic neoadjuvant chemotherapy before radical cystectomy. Induction chemotherapy with MCV before the concurrent cisplatin and radiotherapy was examined. Similar 5-year bladder intact survival rate was noted, with no advantage to induction chemotherapy in phase III evaluation ${ }^{[20]}$. The efficacy of neoadjuvant or adjuvant GC regimens in trimodality therapy remains unknown.

The treatment protocol is considered subjectively well tolerated and this reported by Kevin et al ${ }^{[25]}$. Grades 3 were reported as, leucopenia at $5 \%$, thrombocytopenia at $8 \%$, cystitis in $3 \%$, and hepatic toxicity at $5 \%$. This toxicity profile was comparable to what reported by Elizabeth et al ${ }^{[11]}$, who reported G3 toxicity as, transient elevated liver function at $12 \%$, diarrhea in $8 \%$, hematological in $8 \%$. RTOG $95-06^{[15]}$ reported heamatological G3 toxicity in $18 \%$, cystitis in $6 \%$, and diarrhea in $15 \%$, and that mostly explained by accelerated hyperfractionated radiotherapy. The late toxicity grade 3 reported only in one patient (3\%) developed cystitis ,on the other hand, G3 reported by Donald et al ${ }^{[15]}$ in $12 \%$ heamatological, $6 \%$ cystitis , and $6 \%$ diarrhea. it is related to the different chemotherapy.

\section{Conclusions}

The Tri-modality bladder-sparing approach consists of transurethral resection, chemotherapy twice weekly using gemcitabine and radiotherapy. It may be considered as a reasonable alternative to cystectomy. Also, the approach is preserving the native bladder. Bladder function was preserved in more than $80 \%$ of patients.

The addition of adjuvant chemotherapy is recommended to be addressed in the following studies of bladder preservation in order to reduce the distant failure and improve disease control and the overall survival.

\section{References}

[1] Jason A. Efstathiou, Anthony L. Zietman, John J. Coen, Clinical Radiation Oncology, bladder cancer. 2012; 3rd edition: 1216-1241.

[2] A. Rincón Mayans, D. Rosell Costa, J.J. Zudaire Bergera, et al. Response and progression-free survival in T2 to T4 bladder tumors treated with trimodality therapy with bladder preservation: Spainactas urol esp. 2010; 34(9): 775-780.

[3] Koga F, Yoshida S, Tatokoro M, et al. ErbB2 and NFkB Overexpression as Predictors of Chemoradiation Resistance and Putative Targets to Overcome Resistance in Muscle-Invasive Bladder Cancer: PLoS ONE. 2011; 6(11): 1-5. PMid:22102915 http://dx.doi.org/10.1371/journal.pone.0027616

[4] Choudhury A, Ric Swindell, John P. Loguae, P. Anthony Elliott, Jacqueline E. Livsey, Marcus Wise, Paul Symonds, James P. Wylie, Vijay Ramani, Vijay Sangar, et al. Phase II Study of Conformal Hypofractionated Radiotherapy With Concurrent Gemcitabine in Muscle-Invasive Bladder Cancer. J Clin Oncol. 2011; 29: 733-738. PMid:21205754 http://dx.doi.org/10.1200/JCO.2010.31.5721

[5] Kotwal S, Choudhury A, Johnston C, et al: Similar treatment outcomes for radical cystectomy and radical radiotherapy in invasive bladder cancer treated at a United Kingdom specialist treatment center. Int J Radiat Oncol Biol Phys. 2008; 70: 456-463. PMid:17904301 http://dx.doi.org/10.1016/j.ijrobp.2007.06.030

[6] Stenzl A, Cowan N, De Santis M, et al: The updated EAU guidelines on muscle-invasive and metastatic bladder cancer. Eur Urol. 2009; 55: 815-825. PMid:19157687 http://dx.doi.org/10.1016/j.eururo.2009.01.002

[7] Von der Maase H, Sengelov L, Roberts J, et al: Long-term survival results of a randomized trial comparing gemcitabine plus cisplatin, with methotrexate, vinblastine, doxorubicin, plus cisplatin in patients with bladder cancer. J Clin Oncol. 2005; 23: 4602- 4608. PMid:16034041 http://dx.doi.org/10.1200/JCO.2005.07.757 
[8] Sangar VK, McBain CA, Lyons J, et al: Phase I study of conformal radiotherapy with concurrent gemcitabine in locally advanced bladder cancer. Int J Radiat Oncol Biol Phys. 2005; 61: 420-425. PMid:15667962 http://dx.doi.org/10.1016/j.ijrobp.2004.05.074

[9] Sangar VK, Cowan R, Margison GP, et al: An evaluation of gemcitabines differential radiosensitising effect in related bladder cancer cell lines. Br J Cancer. 2004; 90: 542-548. PMid:14735206 http://dx.doi.org/10.1038/sj.bjc.6601538

[10] Bea Pauwels, Annelies E.C. Korst, Filip Lardon, Jan B. Vermorken. Combined Modality Therapy of Gemcitabine and Radiation. The Oncologist. 2005; 10: 34-51. PMid:15632251 http://dx.doi.org/10.1634/theoncologist.10-1-34

[11] Elizabeth Kent, Howard Sandler, James Montie, et al. Combined-Modality Therapy with Gemcitabine and Radiotherapy as a Bladder Preservation Strategy;results of phase 1 study: Journal of clinical oncology. 2004; 22: 2540-2545. PMid:15226322 http://dx.doi.org/10.1200/JCO.2004.10.070

[12] Common Toxicity Criteria Version 3 [Internet]. Available from: http://ctep.cancer.gov/protocolDevelopment/electronic_applications/docs/ctcaev3.pdf.

[13] Kaufman DS, Winter KA, Shipley WU, et al. Phase I-II RTOG study (99-06) of patients with muscle-invasive bladder cancer undergoing transurethral surgery, paclitaxel, cisplatin, and twice-daily radiotherapy followed by selective bladder preservation or radical cystectomy and adjuvant chemotherapy: Urology. 2009; 73: 833-887. PMid:19100600 http://dx.doi.org/10.1016/j.urology.2008.09.036

[14] Hamza Abbas, Mohamed Aboziada, Mostafa Abdalwanis, et al. Organ Preservation in Bilharzial Bladder Cancer in Egypt: Single Institutional Experience: Journal of Cancer Therapy. 2012; 3: 57-63. http://dx.doi.org/10.4236/jct.2012.31008

[15] Donald s. Kaufman, kathryn a. Winter, william u. Shipley, et al. The Initial Results in Muscle-Invading Bladder Cancer of RTOG 95-06. Phase I/II Trial of Transurethral Surgery Plus Radiation Therapy with Concurrent Cisplatin and 5-Fluorouracil Followed by Selective Bladder Preservation or Cystectomy Depending on the Initial Response: The Oncologist. 2000; 5: 471-476.

[16] Aboziada Mohamed A., Hesham M. Hamza, Ahmed M. Abdelrahem. Initial Results of bladder preserving approach by chemo-radiatiotherapy in patients with muscle invading transitional cell carcinoma: Journal of the Egyptian Nat. Cancer Inst. 2009 June; 21(2): 167-174.

[17] Shipley WU, Kaufman DS, Zehr E, et al. Selective bladder preservation by combined modality protocol treatment: long-term outcomes of 190 patients with invasive bladder cancer. Urology. 2002; 60: 62-7. http://dx.doi.org/10.1016/S0090-4295(02)01650-3

[18] Sauer R, Birkenhake S, Kuhn R, Wittekind C, Schrott KM, Martus P. Efficacy of radiochemotherapy with platin derivatives compared to radiotherapy alone in organ-sparing treatment of bladder cancer. Int J Radiat Oncol Biol Phys. 1998; 40 : 121-7. http://dx.doi.org/10.1016/S0360-3016(97)00579-8

[19] Almudena Zapatero, Carmen Martin de Vidales, Ramón Arellano, et al. Updated results of bladder sparing trimodality approach for invasive bladder cancer. Urologic Oncology: Seminars and Original Investigations (2009).

[20] Shipley WU, Winter KA, Kaufman DS, et al. Phase III trial of neoadjuvant chemotherapy in patients with invasive bladder cancer treated with selective bladder preservation by combined radiation therapy and chemotherapy: Initial results of Radiation Therapy Oncology Group 89-03. J Clin Oncol. 1998; 16: 3576-3583.

[21] Jaewoo Cheon, Hyunchchul Chung, Jaemann Song, et al. Efficacy of Bladder-Preserving Therapy for Patients with T3b,T4a,andT4b Transitional Cell Carcinoma of the Bladder: Korean Journal of Urology. 2010; 51: 525-530. PMid:20733957 http://dx.doi.org/10.4111/kju.2010.51.8.525

[22] Lin CC, Hsu CH, Cheng JC, et al. Induction cisplatin and fluoroucil-based chemotherapy followed by concurrent chemoradiation for muscle -invasive bladder cancer:Int J Radiat Oncol Biol Phys. 2009; 75(2): 442-8. PMid:19307067 http://dx.doi.org/10.1016/j.ijrobp.2008.11.030

[23] Langsenlehner T, Doller C, Quehenberger F, et al. Treatment results of radiation therapy for muscle- invasive bladder cancer: Strahlenther Onkol. 2010 Apr; 186(4): 203-9. PMid:20354664 http://dx.doi.org/10.1007/s00066-010-2053-1

[24] Maarouf AM, Khalil S, Salem EA, et al. Bladder Preservation multimodality therapy as an alternative to radical cystectomy for treatment of muscle invasive bladder cancer: BJU Int. 2011; 107(10): 1605-10. PMid:20825396 http://dx.doi.org/10.1111/j.1464-410X.2010.09564.x

[25] Kevin S Oh, Soto DE, Smith DC, et al. Combined-modality therapy with gemcitabine and radiation therapy as a bladder preservation strategy: long-term results of a phase I trial: Int J Radiat Oncol Biol Phys. 2009 Jun 1; 74(2): 511-7. PMid:18977098 http://dx.doi.org/10.1016/j.ijrobp.2008.08.021 